\title{
Generating concentration time series for simulation studies of hazardous plumes
}

\author{
Ajith Gunatilaka $^{\text {a }}$, Alex Skvortsov ${ }^{\text {a }}$, and Ralph Gailis ${ }^{\mathrm{a}}$ \\ ${ }^{a}$ DSTO, HPP Division, 506 Lorimer Street, Fishermans Bend, Vic 3207, Australia \\ Email: ajith.gunatilaka@dsto.defence.gov.au
}

\begin{abstract}
The threat of chemical, biological, and radiological (CBR) attacks by terrorists against defence forces and civilians has attracted the interest of CBR defence. Major defence purchase decisions in CBR defence are often supported by military operations analysis studies using computer simulations because of safety risks and high cost of field trials involving releases of hazardous CBR agents. Simulation environments such as Hazard Prediction and Assessment Capability (HPAC), developed by Defence Threat Reduction Agency (DTRA), USA, and Chemical, Biological, and Radiological Virtual Battlespace (CBR-VB), developed by Dstl, UK, allow one to simulate releases of CBR agents, forecast their spread, and estimate likely human exposure levels and casualties (Fig. 1). While these simulation packages are capable of simulating the mean concentration fields due to releases of CBR agents, they do not simulate random realisations of concentration fluctuations at present. The ability to generate not only the mean concentrations but also the concentration fluctuations in these environments is crucial if they are to be used to test and evaluate CBR data fusion algorithms, sensor models, CBR sensor network topologies, and models of protective ensembles. The availability of random realisations of concentration fluctuations may also allow more accurate estimation of human health effects of CBR agents in different threat scenarios.
\end{abstract}

The literature describes ongoing efforts at generating such random realisations using computational fluid dynamic modelling, Lagrangian particle simulation, etc. While these approaches are capable of generating random plume realisations when reliable and detailed input data is available, they are time consuming and require expert knowledge and advanced computer resources. This paper describes a simple approach we have developed to generate realistic concentration fluctuation time series for HPAC and CBR-VB simulation studies when a complete plume realisation over the whole spatial domain is not required and random concentration fluctuation time series at selected locations is sufficient. This approach uses mean concentra-

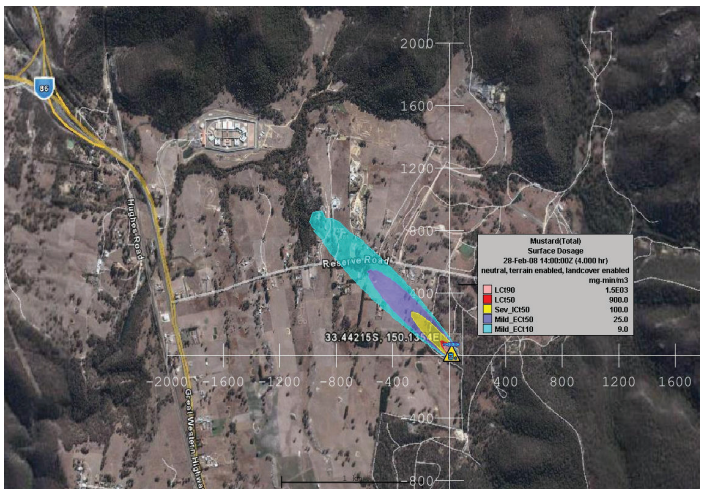

Figure 1. Output of an HPAC simulation tion, concentration variance, and correlation time scale data, which are available from the simulation environment, to determine the parameters of the likely probability density function (PDF) of concentration fluctuations at a given point within a simulated plume. Assuming that the concentration fluctuations can be characterised by a left-shifted and clipped gamma distribution, the parameters of this PDF are determined by solving a system of transcendental equations. Once the PDF is determined, a numerical stochastic model is employed to generate concentration time series assuming that each time series obeys a first order Markov process and that the concentration time series are non-intermittent and stationary. These non-intermittent time series are baseline shifted and clipped to simulate concentration time series with intermittent zeros. In this paper, we discuss the theoretical basis that underpins our approach and some implementation aspects. We also outline how this method can be extended to cases where the stationarity assumption does not hold.

Keywords: Concentration fluctuations, stochastic time series model, simulation environment 


\section{INTRODUCTION}

The threat of chemical, biological, and radiological (CBR) attacks has attracted the interest of CBR defence. Because of safety risks and high cost of field trials involving releases of hazardous CBR agents, computer simulations are widely used for military operations analysis studies carried out to support major purchase decisions in CBR defence (for example, large scale acquisitions of CBR protection ensembles, sensors and sensor networks, and warning and reporting systems). Simulation environments such as Hazard Prediction and Assessment Capability (HPAC), developed by Defence Threat Reduction Agency, USA, and CBR Virtual Battlespace (CBR-VB), developed by Dstl, UK, allow one to simulate releases of CBR agents, forecast their spread, and estimate likely human exposure levels and casualties. While these simulation packages are capable of simulating the mean concentration fields due to releases of CBR agents and providing an estimate of concentration variances, they cannot simulate concentration fluctuation realisations at present. The ability to generate not only the mean concentrations and variances but also realisations of concentration fluctuations in these environments is crucial if they are to be used to test and evaluate CBR data fusion algorithms, CBR sensor models, sensor network topologies, and models of CBR protective ensembles.

Some operations analysis studies require concentration time series measured at fixed locations (for example, when simulating measurements collected by a fixed sensor) or along a particular path of travel (for example, when simulating the hazardous exposure of a soldier moving along the path)(Hill and Mitchell (2009)). In HPAC and CBR-VB, fixed or moving samplers can be specified for this purpose. However, the time series acquired by these samplers are smooth time series that approximate ensemble averages of a large number of random concentration realisations. Health effects caused by exposure to a concentration realisation that contains intermittent fluctuations may be quite different to those caused by exposure to the corresponding ensemble average concentration over the same duration (Ride (1995); Hilderman et al. (1999); Yee (1999)).

Some recent studies compare estimates of health hazards computed using ensemble averaged plumes that are traditionally available from atmospheric transport and dispersion codes against estimates obtained using random realisations generated using alternative approaches. Long et al. (2009) compare a hypothetical chlorine release modelled using HPAC against the same release modelled using computational fluid dynamics (CFD). While plume footprints of the two methods were found to be in good agreement with each other, the maximum concentrations in the CFD simulations were found to be higher than those of the HPAC simulations. Bogen and Gouveia (2008) used a statistical simulation assessment method, calibrated to data from a large-scale experimental field trial, to assess the combined impact of spatiotemporal fluctuation on the size of assessed threat areas. They found that standard methods for assessing hazard areas that rely on predicted mean concentrations and acute exposure guideline levels (AEGL) seriously underestimate the size of hazard areas. Czech et al. (2011) compared hazard area predictions based on individual plume realisations to those based on ensemble mean plumes; for chemicals that have toxic exponents greater than one, hazard areas derived from ensemble mean averages were found to be significantly smaller than those derived from typical plume realisations.

Apart from underestimating human health impacts, the ensemble mean concentrations could also lead to unreliable outcomes in operations analysis studies that investigate the performance of CBR sensors and networks, warning and reporting systems, and protective ensembles. Therefore, it is crucial to develop means for generating realistic concentration fluctuation data for quantitative CBR operations research studies; different approaches being pursued for this purpose can be found in the literature. For example, the National Center for Atmospheric Research (NCAR) is developing the Virtual THreat Response Emulation and Analysis Testbed (VTHREAT) modelling system, which uses large eddy simulation coupled with a Lagrangian particle dispersion model (Bieberbach et al. (2010)); concentration realisations generated using VTHREAT were used in the aforementioned study by Czech et al. (2011). Another approach for generating concentration realisations is the use of computational fluid dynamics (CFD) simulation; for example, Long et al. (2009) used CFD to generate concentration realisations for their study.

While the approaches described above are capable of generating concentration fluctuation realisations when reliable and detailed input data are available, they are time consuming and require expert knowledge and advanced computer resources; for example, according to Long et al. (2009), their CFD simulations, 
run on eight nodes of a Beowulf computer cluster, required 200 hours to simulate six minutes of the chemical release.

In this paper, we describe a simple approach we have developed to generate random realisations of concentration fluctuations for operations analysis studies. This approach leverages information that is readily available from a standard atmospheric transport and dispersion model to generate concentration realisations corresponding to points of interest within the simulation domain. The proposed approach may be used when a full concentration realisation over the whole spatial domain is not necessary for a particular study and random concentration time series at selected sampler locations is sufficient.

The paper is organised as follows. We describe the theoretical basis of our approach in Section 2. A description of the implementation and some results are presented in Section 3. Conclusions are drawn and future work outlined in Section 4.

\section{TheOretiCAL BASIS}

The concentration in a hazardous chemical plume dispersing in the atmosphere is inherently a random variable because of the stochastic nature of the underlying turbulent dispersion processes. Therefore, various statistical moments, such as mean and variance, are typically used to characterise these random concentration fluctuations. Because all statistical moments can be found if the probability density function (PDF) of a random quantity is known, the concentration PDF provides a convenient means to summarise the statistical characteristics of random concentration fluctuations. A number of PDFs, including clipped normal (Lewellen and Sykes (1986)), lognormal (Yee et al. (1993)), exponential (Hanna (1984)), and clipped gamma (Yee and Chan (1997); Yee (2009)), have been proposed in the past to characterise concentration fluctuations of dispersing plumes.

Yee and Chan (1997) and Yee (2009) showed that the left-shifted and clipped gamma PDF can well approximate the measured probability distribution of concentration for plumes dispersing in built-up environments as well as in open and unobstructed terrain. More importantly, they showed that it is possible to obtain the parameters of this PDF when only the mean concentration and normalised mean square concentration are known. Because mean concentration and concentration variance at any location within a simulated dispersing plume are available from HPAC and CBR-VB, we can compute the normalised mean concentration and, hence, determine the left-shifted and clipped gamma PDF suitable for characterising concentration fluctuations at these locations. Once the PDF is determined, we can generate random realisations of data to mimic concentration fluctuations corresponding to the chosen locations.

\subsection{Probabilistic model of concentration fluctuations}

The left-shifted and clipped gamma PDF chosen for characterising the concentration has the following form (Yee (2009)):

$$
f(c ; \mathbf{x})=\left(\frac{c+\lambda}{s}\right)^{k-1} \frac{\exp (-(c+\lambda) / s)}{s \Gamma(k)}+(1-\gamma) \delta(c),
$$

with $k=k(\mathbf{x})>0, s=s(\mathbf{x})>0, \lambda=\lambda(\mathbf{x}) \geq 0, \gamma=\gamma(\mathbf{x}) \in[0,1]$. Here, $k$ and $s$, respectively, are the shape parameter and the scale parameter of the gamma distribution, and $\lambda$ is the left shift. $\Gamma(x)$ is the gamma function, $\delta(x)$ is the Dirac delta function, and $\gamma$ is the intermittency factor that models the probability of observing a non-zero instantaneous concentration at $\mathbf{x}$. Concentration $c$ has the range $c \in[0, \infty)$. While the clipped gamma PDF of (1) has four parameters: $k, s, \lambda$, and $\delta$, Yee and Chan (1997) showed that only three of these are independent. To determine the parameters of the PDF of the normalised concentration $\chi / C$, where $\chi$ is the instantaneous concentration and $C$ the mean concentration, they derived the following system of transcendental equations:

$$
\begin{aligned}
& \frac{1}{s}=\left(-\frac{\lambda}{s}+k\right) \gamma+\frac{1}{\Gamma(k)}\left(\frac{\lambda}{s}\right)^{k} \exp (-\lambda / s) \\
& \left\langle\left(\frac{\chi}{C}\right)^{2}\right\rangle=\frac{((\lambda / s) \gamma+(-\lambda / s+k+1) / s)}{\left[(-\lambda / s+k) \gamma+(\lambda / s)^{k} \exp (-\lambda / s) / \Gamma(k)\right]^{2}}
\end{aligned}
$$




$$
\gamma=\min \left(1,3\left\langle\left(\frac{\chi}{C}\right)^{2}\right\rangle^{-1}\right)=\frac{\Gamma(k ; \lambda / s)}{\Gamma(k)}
$$

From the first equality of (4), $\gamma$ can be computed as a function of the normalised mean square concentration. Then, the system of transcendental equations can be solved numerically for $k, s$ and $\lambda$.

\subsection{Stochastic time series model}

After obtaining the parameters that define a concentration fluctuation PDF, as described in Section 2.1, we need a technique to generate random realisations of fluctuations from that PDF. For this purpose, we consider a stochastic time series model, developed by Du et al. (1999), which can generate a time series that mimics the concentration time series at a point within a dispersing plume. This model assumes that the concentration time series at a given spatial point is continuous, with no intermittent zero concentration intervals. Then, the evolution of the concentration time series is represented as a first-order Markov process given by the stochastic differential equation (Du et al. (1999)):

$$
d c=a(c, t) d t+b(c, t) d \zeta
$$

where $a(c, t)$ is the drift coefficient, $b(c, t)$ is the diffusion coefficient, and $d \zeta$ is a Wiener process with variance $d t$ and zero mean.

Functions $a(c, t)$ and $b(c, t)$ are related to each other through the Fokker-Planck equation:

$$
\frac{\partial \rho_{c}}{\partial t}=-\frac{\partial}{\partial c}\left(a \rho_{c}\right)+\frac{1}{2} \frac{\partial^{2}}{\partial c^{2}}\left(b^{2} \rho_{c}\right)
$$

where $\rho_{c}$ is the PDF of concentration fluctuations. In the case of a steady plume, for which $\frac{\partial \rho_{c}}{\partial t}=0$ holds, it is possible to obtain a simple constraint that relates $a(c, t)$ and $b(c, t)$.

For $a$, Du et al. chose:

$$
a=-\frac{c-C}{T},
$$

which forces the concentration fluctuations towards the mean value $C$. By plugging in $a$ from (7) in the Fokker-Planck constraint equation, (6), and integrating, they derived the following expression for $b^{2}$ :

$$
b^{2}=\frac{2}{T \rho_{c}(c)} \int_{c}^{\infty}(c-C) \rho_{c}(c) d c
$$

To find the appropriate $b^{2}$ for our simulations, we substitute in (8) the expression for a gamma PDF, shown in (9)

$$
f(c ; \mathbf{x})=\left(\frac{c}{s}\right)^{k-1} \frac{\exp (-c / s)}{s \Gamma(k)},
$$

which results in:

$$
b^{2}=\frac{2}{T \rho_{c}(c) \Gamma(k)}\left[s \Gamma\left(k+1, \frac{c}{s}\right)-C \Gamma\left(k, \frac{c}{s}\right)\right] .
$$

Once expressions for $a$ and $b$ are known, the stochastic model is obtained by discretising the stochastic differential equation, as (Du et al. (1999))

$$
c_{n+1}=c_{n}+a\left(c_{n}\right) \Delta t+b\left(c_{n}\right) \sqrt{\Delta t} m_{n},
$$

where $\Delta t$ is the simulation time step, $c_{n}$ is the instantaneous concentration at time $t_{n}=n \Delta t$, and $m_{n}$ is a Gaussian random number with zero mean and unit variance. 


\subsection{Simulating intermittent time series}

Because inhomogeneous mixing of a pollutant in air can cause instantaneous concentrations that range from zero to many times the mean concentration, it is important to be able to simulate such intermittent time series accurately. The stochastic time series model proposed by Du et al. for simulating continuous, non-intermittent concentration fluctuations was extended by Hilderman and Wilson (1999) to allow simulation of intermittent concentration time series containing periods of zero concentration. While Hilderman and Wilson (1999) considered a shifted and clipped log normal PDF to describe concentration fluctuations, we apply their approach to a shifted and clipped gamma PDF.

To simulate intermittent concentration time series data using this extended stochastic model, we first generate continuous, non-intermittent, and non-negative data by using the appropriate gamma PDF parameters, $k$ and $s$, and correlation time $T_{c}$. Following the notation of Hilderman and Wilson (1999), we denote these non-negative data by $c_{+}$. A segment of such a time series, normalised by its mean value $C_{+}$, is shown in Figure 2. Next, we shift the PDF by subtracting $\lambda$ from $c_{+}$. This step, which is referred to as baseline shifting in Hilderman and Wilson (1999), produces a new time series, denoted by $\tilde{c}$, with some negative values (Fig. 3 ) and mean $\tilde{C}$. Finally, all negative values of $\tilde{c}$ are clipped and replaced by zeros to generate the desired intermittent concentration time series $c$ (Fig. 4), with mean value $C$. It should be noted that clipping does not affect the non-negative parts of $\tilde{c}$, and the reason why the peak value of the concentration time series in Figure 4 appears to be smaller than that in Figure 3 is only because the figures show concentrations normalised by their respective means, with $C>\tilde{C}$.

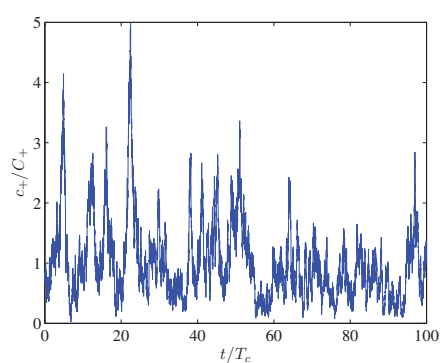

Figure 2. Unshifted nonintermittent time series

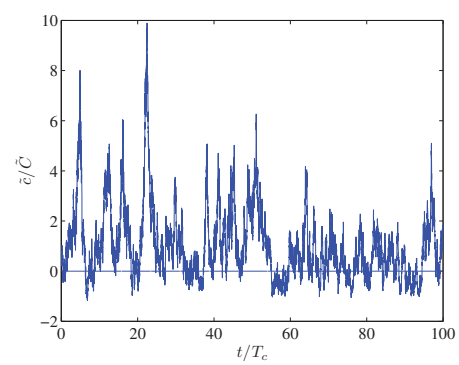

Figure 3. Baseline shifted time series

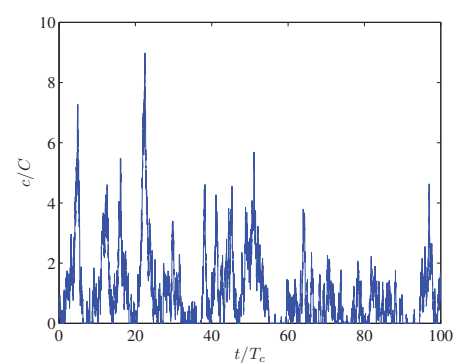

Figure 4. Shifted and clipped time series

\section{IMPLEMENTATION AND RESULTS}

Using the theoretical basis described in Section 2, we can proceed to generate time series data that mimic concentration fluctuations at selected points within a simulated hazardous plume. In this section, we describe how the proposed approach is implemented to generate concentration time series corresponding to locations within a plume simulated in HPAC.

The first step of the method is to create a sampler input file to specify sampling locations within the HPAC simulation spatial domain at which we desire to extract concentration mean, variance, and correlation time. A portion of such a sampler input file, specifying the longitudes and latitudes of a number of samplers for sampling the concentration of chemical warfare agent GB, is shown in Figure 5 (a).

Next, HPAC is run to simulate atmospheric transport and dispersion of the source material. Figure 6 shows the plume generated by HPAC when a hypothetical release of GB was simulated. The red dots shown on this figure denote the locations of samplers specified in the sampler input file. When HPAC's sampling option is enabled, the simulation produces an output file, similar to the one shown in Figure 5 (b), that lists the mean concentration, concentration variance, and correlation time at each of the samplers, sampled at a user-specified output interval. If $N$ samplers are specified in the input file, then the sampler output file will have $3 N+1$ columns, with the first column listing the output time (T), and the remaining columns listing mean concentration, concentration variance, and correlation time, respectively, for each of the $N$ samplers. Column headings (C00n), (V00n), and (T00n) identify the mean concentration,concentration variance, and correlation time at the $n$th sampler, where $n=1,2, \ldots, N$. 


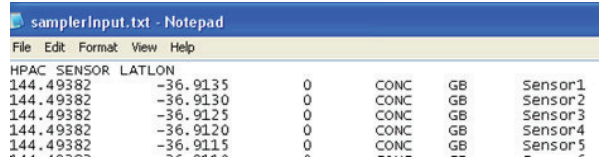

(a)

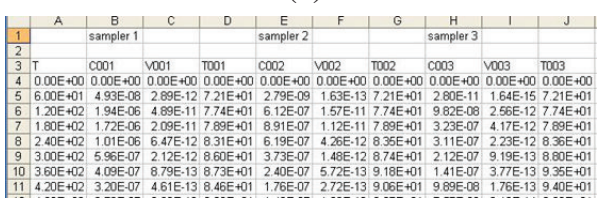

(b)

Figure 5. Typical HPAC sampler input and output files

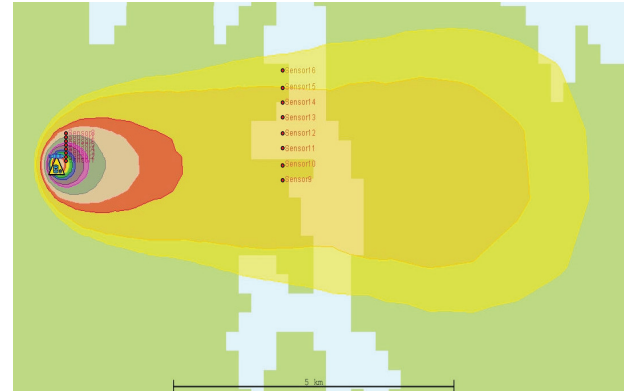

Figure 6. Hypothetical HPAC plume overlaid with sampler positions

Let $C_{n}(k), V_{n}(k)$, and $T_{n}(k)$ be the mean concentration, variance, and correlation time, respectively, of the $n$th sampler at the $k$ th sampling instant. If the plume is steady, stationarity will hold and these values will be independent of $k$. For now, we assume stationarity and let $C_{n}(k)=C_{n}, V_{n}(k)=V_{n}$, and $T_{n}(k)=T_{n}$. Next, we compute the normalised mean square concentration at the $n$th sampler as

$$
\left\langle\left(\frac{\chi}{C}\right)^{2}\right\rangle=\frac{V_{n}}{C_{n}^{2}}+1 .
$$

By plugging in the computed normalised mean square concentration in the first inequality of (4), we compute the intermittency $\gamma$ at the sampler location. Then from the numerical solution of the system of trancendental equations of (2), (3), and (4), we compute $k, s$, and $\lambda$ parameters that define the PDF of normalised concentration at the sampler. Next, by applying the stochastic time series model, we generate a continuous, nonintermittent time series. This time series is then shifted by $\lambda$ and clipped to obtain a time series with intermittent zeros. Because this resulting time series is a normalised concentration, with the mean equal to one, it is then scaled by mean concentration $C_{n}$ to obtain the final concentration time series with target statistical characteristics. Figure 7 shows a concentration realisation generated using this approach for a sampler location

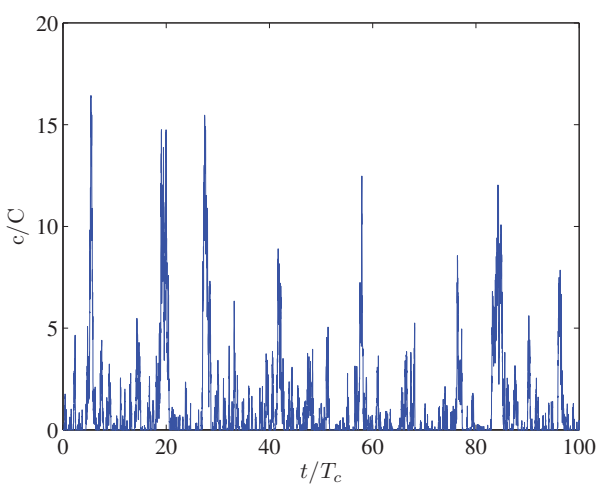
within the simulated GB plume of Figure 6.

Many practical hazardous agent release scenarios simulated using HPAC do not satisfy the stationarity conditions assumed in the previous section. Therefore, it is important to extend the approach developed above to handle the non-stationary case. In the non-stationary case, we cannot assume $C_{n}(k), V_{n}(k)$, and $T_{n}(k)$ to be the same at all times. However, if we use a small enough output interval for HPAC samplers so that the rate of change of concentration during each interval is small, we may assume stationarity to approximately hold within each sampling interval. This piecewise stationary assumption will be used to apply the approach developed for the stationary case to each of the sampling intervals enabling us to generate concentration realisations for non-stationary release scenarios.

\section{Conclusions}

In this paper, we presented an approach for generating realistic concentration fluctuation time series that are consistent with mean concentration, concentration variance, and correlation time scale information 
available from a standard atmospheric transport and dispersion model. Assuming these concentration time series to have a clipped-gamma PDF, this approach estimates the parameters of PDFs at points of interest within a plume simulated in the dispersion model. Random realisations of concentration fluctuation time series corresponding to these positions are generated using a stochastic time sereis model.

While the paper presented how concentration fluctuation time series are generated in the stationary case, where simulation is of a steady source emitting into a statistically stationary flow, it is currently being extended to the non-stationary case. At present, the proposed approach also assumes sampler locations within the simulated plume as fixed. We hope to extend our approach to include mobile samplers in future, and this extension will enable the estimation of toxic loads experienced by people moving within a hazardous plume environment.

\section{REFERENCES}

Bieberbach, G., P. E. Bieringer, P. E. Wyszogrodzki, et al. (2010). Virtual chemical and biological (CB) agent data set generation to support the evaluation of $\mathrm{CB}$ contamination avoidance systems. In The Fifth Symposium on Computational Wind Engineering (CWE 2010), Chapel Hill, North Carolina.

Bogen, K. T. and F. J. Gouveia (2008). Impact of spatiotemporal fluctuations in airborne chemical concentration on toxic hazard assessment. Journal of Hazardous Materials 152, 228-240.

Czech, C., N. Platt, J. Urban, et al. (2011, January). A comparison of hazard area predictons based on the ensemble-mean plume versus individual plume realizations using different toxic load models. In 91st American Meteorological Society Annual Meeting, Washington, USA.

Du, S., D. J. Wilson, and E. Yee (1999). A stochastic time series model for threshold crossing statistics of concentration fluctuations in non-intermittent plumes. Boundary-Layer Meteorology 92, 229-241.

Hanna, S. R. (1984). The exponential probability density function and concentration fluctuations in smoke plumes. Boundary-Layer Met 29, 361-375.

Hilderman, T. L., S. E. Hrudey, and D. J. Wilson (1999). A model for effective toxic load from fluctuating gas concentrations. Journal of Hazardous Materials 64, 115-134.

Hilderman, T. L. and D. J. Wilson (1999). Simulating concentration fluctuation time series with intermittent zero periods and level dependent derivatives. Boundary-Layer Meteorology 91, 451-482.

Hill, A. M. and I. R. Mitchell (2009, July). Quantitative modelling of the performance of potential australian chemical, biological \& radiological defence systems - a pilot study. In 18th World IMACS / MODSIM Congress, Cairns, Australia, pp. 1615-1621.

Lewellen, W. S. and R. I. Sykes (1986). Analysis of concentration fluctuations from lidar observations of atmospheric plumes. J. Clim. appl. Met 85, 1145-1154.

Long, K. J., R. J. Zajaczkowski, S. E. Haupt, and L. J. Peltier (2009). Modelling a hypotheitical chlorine release on a college campus. Journal of Computers 4, 881-890.

Ride, D. (1995). A practical method of estimating toxic loads in the presence of concentration fluctuations. Environmetrics 6, 643-650.

Yee, E. (1999). An impact-effect mathematical model incorporating the influence of exposures to fluctuating concentrations in a dispersing plume of pollutants in the atmosphere. J Expo Anal Environ Epidemiol 9, 300-311.

Yee, E. (2009). Probability law of concentration in plumes dispersing in an urban area. Environ Fluid Mech 9, 389-407.

Yee, E. and R. Chan (1997). A simple model for the probability density function of concentration fluctuations in atmospheric plumes. Atmos Environ 31, 991-1002.

Yee, E., P. R. Kosteniuk, G. M. Chandler, C. A. Biltoft, and J. F. Bowers (1993). Statistical characteristics of concentration fluctuations in dispersing plumes in the atmospheric surface layers. Boundary-Layer Met 65, 69-109. 